\title{
Author Index Vol. 17,1997
}

Abe,K. 137 Abed,J. 118 Abu-Aisha,H. 118 Adabra,Y. 17 Aguiari, G. 458 Ahuja,T.S. 480

Akiyama, K. 25 Akmal,M. 12 AlDesouki,M. 118 Al-Harbi,N. 68 Al-Homrany, M. 32 AlMohannadi, Sh. 505 Al-Mohaya,S. 495 Al-Wakeel,J. 118,495 Alexiewicz, J.M. 12 Aliotta,G. 241 Anaya,F. 487 Andreae, L. 359 Andres, A. 445 Angeletti, L.R. 228 Angelopoulos, N. 214 Antonello,A. 124, 172,248 Arakawa, M. 499 Aranda, J.L. 445

Bagnis,C. 17 Baluarte, H.J. 146 Bang,B.K. 42,483 Baran,D. 528 Bastani,B. 474 Basu,S. 59

Bellini, L. 318 Bercovitch, D. 477 Berridge, MJ. 1 Better, O.S. 392 Bhattacharyya, N. 435

Bisaccia, C. 318 Boice,J.L. 89 Bonfante,L. 172,248 Bonomini,V. 274 Bordin, V. 172,248

Borsatti,A. 172 Bozza,A. 458 Brunati,C. 103

Calò,L. 124,172,203,248 Cameron, J.S. 347 Campieri, C. 274 Cantaro, S. 124 Cantarovich, M. 450 Cavarra, B. 228 Cazabat, M. 417

Chabannier, M.-H. 46, 489 Chamma,F. 399 Chan,J.C.N. 72 Chan,T.Y.K. 72 Chang, C.-C. 193 Chang, Y.S. 42 Charlet, J.-P. 417 Chen,T.W. 158 Cheng, C.-H. 541 Cheng, F.-C. 541 Cheung, A.H.S. 435 Choi,EJ. 42 Choi,YJ. 42 Chuang,L.-Y. 193 Chung, D.R. 112 Chung, Y.-H. 112 Cisterne, J.-M. 46,489 Cockram,C.S. 72 Colussi,G. 103 Critchley, J.A.J.H. 72 Crosson, A.W. 176 Cruz,D.N. 181

D'Angelo,A. 124,172,248

Dal Canton, A. 282

Dal Canton, I. 282

Dang, C.R. 435

Dardioti, V. 214

Dasgupta, M. 524

Davidman, M. 477

Davidson, S.A. 53

DeBroe, M.E. 252

De Ferrari, M.E. 103

De Paoli Vitali, E. 458

De Santo, D. 318

De Santo, L.S. 318

De Santo, N.G. 201,241,261,

318 De Santo, R.M. 318 De Weerdt, D.L. 252 Deforest, A. 146 Del Palacio, A. 445 Denis, A. 399 Deray, G. 17 Deschênes, J. 450 Diamandopoulos, A.A. 222, 304 Distant, D. 425 Dubois, M. 17,417 Duffaut,M. 417 Durand,D. 46,417,489

Eftychiadis, A.C. 217 Eknoyan, G. 201,261 ElGamal,H.H. 495 El-Reshaid, K. 505 El-Reshaid, W. 505 El-Shahawy, M. 511 Eliakim,A. 471 Elisaf, M.S. 153 Escribano,G. 487

Fasoli,G. 124 Favaro,S. 172,248 Federman, M. 176 Fellner, S.K. 289 Fernandez-Miranda, C. 445 Fine,L.G. 359 Flynn,J.T. 146 Freter, S. 477 Friedman, E.A. 425 Frosio-Roncalli, M. 282 Fukushima, M. 382 Fulop,M. 421 Futrakul,N. 533 Futrakul,P. 533 
Garza,R. 59 Gejyo,F. 499 Germanos, N.P. 153 Giacomini, A. 172 Gill, S.K. 12 Glicklich,D. 480 Gohh,R.Y. 176 Gomez Campderá, F. 487 Gómez-Gerique, J. 445 Gottschalk, C.W. 289 Goya,N. 440 Guh,J.-Y. 36,193 Guijarro, C. 445

Hadjiconstantinou, V. 214 Hansberry, K.L. 96 Hazani, E. 233 Hewan-Lowe, K. 462 Hicks, J. 347 Hierholzer, J. 369 Hierholzer, K. 369 Higuchi,T. 428 Hirata, K. 25 Homel,P. 425 Hong,J. 425 Huang, T.-P. 158

\section{KAílGEH}

(C) 1997 S. KargerAG, Basel

E-Mail karger@karger.ch Fax + 41613061234 http://www. karger. ch Huraib, S. 118 Hyppolite, J.C. 425

Icart,J. 46,489 Ifudu,O. 425 Iguchi, Y. 440 Inoue, T. 382 Iorio, L. 286 Ishikawa, N. 440 Ishimura, E. 382 Izopet, J. 46,417

Jaar,B. 399 Jacobs, C. 17 Jacquiaud, C. 17 Jaqua,R.A. 89 Jaudon,M.C. 17 Jena, M. 462 Jothy,S. 528 Jovanovski, M. 128 Jungthirapanich, J. 533

Kaiser, B.A. 146

Kanmatsuse, K. 428 Kantaropoulou, M. 406 Karagianni, A. 406 Karayalcin, U. 100 Karpinski, J. 528 Kato,K. 81 Kechrid,M.S. 495 Khalil,A. 165 Khan,G.A. 524 Khwaja,S. 511 Kim,B.K. 483 Kim,H.A. 483 Kim,S.B. 112 Kim,S.Y. 42 Kim,Y.K. 483 Kim,Y.S. 42 Kodera, S. 466 Korzets,Z. 471 Koulouridis, E.I. 153 Kramberger, S. 93 Kume,K. 81 Kuno,T. 428 Kyriazis, G. 406

Lai,Y.-H. 36,193 Lee,J.H. 483 Lee, S.H. 42 Lee,Y. 112 Levy,M. 528 Lewis, F.I. 524 Lipman, M. 477 Lireman, D. 68 Long, S.S. 146

Macey,L.J. 425 McNamara, J.J. 435 Makino,H. 25

Malik, G.H. 495

Marcinkowski, W. 187

Marketos, S.G. 201,204,205

Martinet, O. 489

Massry, S.G. 12,187,201,233,511

Matsuda, M. 25

Meani, A. 172

Medina, R. 59

Memon, N. 118

Merouani, A. 53

Mezzogiorno, A. 269

Mezzogiorno, V. 269

Mignon, F. 399

Miorelli,M. 124

Mitch, W.E. 462

Mitwalli, A.H. 495

Mooraki, A. 474

Morales, J.M. 445

Morii,H. 382

Morise,T. 100

Morita,A. 382

Moustafa, F.E. 165 
Mutoh, S. 81

Myers, S.A. 435

Nacca,R.G. 286 Nagy,J. 387 Nakazawa, H. 440 Ng,R,C.K. 435 Nishii,Y. 382 Nishiki,T. 413

Nistri, S. 124 Normanno, M. 172 Nosrati, S.M. 511 Nzerue, Ch. 462

Oda,H. 413 Ogawa,T. 413 Ohmuro, H. 466 Ootaka,T. 137 Ota, K. 25, 440 Ota,Z. 25

Palisi,M. 124 Panicali, H. 46 Pappas,M.B. 153 Park,J.S. 112 Pecovnik-Balon, B. 93 Peitzman, S.J. 299 Perini,L. 458 Petrusevska, G. 128 Piomelli,D. 241 Polenakovic, M. 128 Polinsky, M.S. 146 Pollio,A. 241 Pomeranz, A. 471 Pomeranz, M. 471 Porres, A. 445 Poshyachinda, M. 533 Poulakou-Rebelakou, E. 209

Progia,E. 406 Puel,J. 417 Pugh,J.A. 59 Purkerson, M.L. 340

Radoux,V. 528 Richet,G. 315 Rippa-Bonati, M. 248 Rodicio, J.L. 445 Rodriguez, P. 487

Rofougaran, R. 474 Rombolà, G. 103 Rostaing,L. 46,417,489 Rovang, R.D. 89

Sacré,D. 252 Sadikario, S. 128 Saito,T. 137 Santella, R.N. 89 Saso,R. 128 Sato,H. 499

Sayada,C. 417 Scalia,P. 458 Scapoli,C. 458 Schlanger, L. 462 Schrier,R.W. 53 Schulman, S.L. 146 Scognamiglio, R. 124 Senno,L. del 458 Sensirivatana, R. 533 Shafik,A. 518 Shasha,S.M. 233 Shikata,K. 25 Shin, S.-J. 36 Shirato, I. 466 Shu,K.-H. 541 Siamopoulos, K.C. 153 Sikole,A. 128 Simonelli, R. 286 Singklwa,V. 533 Siohan,P. 399 Sitprija,V. 533 Sivanandan, R. 505 Smogorzewski, M. 12, 187, 233 Snelders, E.D. 252 Sobh,M.A. 165 Soderdahl, D.W. 96 Soma, J. 137 Sombolos,K. 406 Sommer,B.G. 425 Sonkodi, S. 387 Stojanovic, A. 128 Sugathan,Th. 505 Sugimoto, H. 25 Sulimani, R.A. 495 Sumrani, N. 425 Suzuki, H. 81 Suzuki, J. 81 Suzuki, S. 81,499

Author Index

Am J Nephrol Vol. 17, 1997

Tabata,T. 382 Takahashi,H. 499 Takahashi, K. 440 Takahashi,S. 428 Takashima, N. 499

Tanabe,K. 440 Taniguchi, Y. 413 Tamg,D.-C. 158 Tashiro, K. 466 Thrasher, J.B. 96 Tokumoto, N. 440 Toma,H. 440 Tomino, Y. 466 Tomlinson, B. 72 Ton-That,H. 417 Touwaide, A. 241

Tsai,J.-H. 36,193 Tsitamidou, Z. 406

Uziel,Y. 471

Valderrabano, F. 487 Verdy,E. 399 Vianello,D. 172 Viron,B. 399

Wahab,M.A. 165 Watana,D. 533 Wechsler,L. 340 Welter, R.L. 89 Williams, M.E. 176

Wolach,B. 471 Wong,L.L. 435 Wu,M.-J. 541

Xue-Feng,Y. 413

Yagisawa, T. 440 Yakupoglu,G. 100 Yamakido, M. 413 Yamashita, K. 413

Yang,C.W. 42,483 Yang,M.-L. 193 Yang,W.S. 112 Yang,Y.-L. 193 Yenrudi,S. 533 Yoon, S.N. 483 Yorioka,N. 413 Yoshida,K. 81 Yu,E. 112 Yu,L.-K. 36 Yusa,A. 137

Zambetti, F.X. 176 Zawada, E.T., Jr. 89 Zerbo,F. 124 Zhang, G. 187 Zingman,B. 480

Zuccoli,M. 274

546

Am J Nephrol Vol. 17, 1997

Author Index 